\title{
Description of Dispersive Wave Emission and Supercontinuum Generation in Silicon Waveguides Using Split-Step Fourier and Runge-Kutta Integration Methods
}

\author{
Xuefeng Li \\ School of Science, Xian University of Post \& Telecommunications, Xian 710121, China \\ Correspondence should be addressed to Xuefeng Li; lixfpost@163.com
}

Received 6 January 2014; Accepted 2 March 2014; Published 27 March 2014

Academic Editor: Boris G. Konopelchenko

Copyright @ 2014 Xuefeng Li. This is an open access article distributed under the Creative Commons Attribution License, which permits unrestricted use, distribution, and reproduction in any medium, provided the original work is properly cited.

Based on solving numerically the generalized nonlinear Schrödinger equation describing the propagation of high order femtosecond soliton in silicon waveguide under certain parametric conditions by the split-step Fourier and Runge-Kutta integration methods, dispersive wave emission and supercontinuum generation in silicon waveguides are numerically investigated by propagating femtosecond solitons. The numerical results show that the efficient dispersive wave emission can be generated in silicon waveguide, which plays an important role in the process of the supercontinuum generation with the form of Cherenkov radiation, and it is also shown that the high order low-energy solitons and short waveguides are efficient for the dispersive wave emission.

\section{Introduction}

Supercontinuum generation has important potential applications in the fields such as optical communication, spectroscopy, and optical coherence tomography [1]. Many researches of supercontinuum generation in different waveguide structures such as single mode fibers [2], photonic crystal fibers (PCFs) [3-5], and silica nanowires [6] have been reported. These results indicate that it is possible to achieve supercontinuum generation at relative low optical power and over short propagation distances provided that the medium exhibits high nonlinear response and tailoring dispersion properties. However, it is difficult to implement on-chip integration applications because of the large propagation length required by the large spectral broadening.

A promising solution to generating supercontinuum is provided by the silicon waveguide, which has the advantage of employing an emerging silicon integrated photonics platform. Compared with the conventional optical waveguides, the silicon waveguide has several unique properties that can be employed to achieve on-chip scale supercontinuum generation. The silicon waveguide has a smaller transverse dimension governing the dispersion properties so that the dispersion properties can be tailored flexibly by designing the transverse dimension properly $[7,8]$. At the same time, the optical intensity used is a moderate input power due to high optical confinement in waveguide caused by the high index contrast; therefore the nonlinear optical effects are enhanced intensely [9-11]. Moreover, the nonlinear coefficient of the silicon waveguide is several orders of magnitude higher than that of the PCFs. These properties make the silicon waveguide significant and suitable for the supercontinuum generation $[12,13]$.

In this paper, through solving numerically the generalized nonlinear Schrödinger equation describing propagation of femtosecond pulse in silicon waveguide under certain parametric conditions by the combination of split-step Fourier and Runge-Kutta integration methods, the dispersive wave emission and supercontinuum generation in silicon waveguide are investigated by propagating femtosecond solitons.

\section{Theory Model}

The theory model that describes the femtosecond pulse propagation along the silicon waveguide including the effects 
of nonlinear loss such as the two-photon absorption (TPA) and free-carrier absorption (FCA) is based on the generalized nonlinear Schrödinger equation as follows [14]:

$$
\begin{aligned}
\frac{\partial A}{\partial z}= & \sum_{m=2}^{\infty} \frac{i^{m+1} \beta_{m}}{m !} \frac{\partial^{m} A}{\partial t^{m}}-\frac{1}{2}\left(\alpha_{l}+\alpha_{f}\right) A+i \gamma \\
& \times\left(1+\frac{i}{\omega_{0}} \frac{\partial}{\partial t}\right) A(z, t) \times \int_{-\infty}^{t} R(t-\tau)|A(z, \tau)|^{2} d \tau
\end{aligned}
$$

where $A=A(z, t)$ is the electric field envelope as a function of distance along the waveguide $z$ and retarded time $t ; \alpha_{l}$ and $\alpha_{f}$ account for the linear losses and the free-carrier absorption (FCA) of the waveguide, respectively; $\beta_{m}$ s are dispersion coefficients at center frequency $\omega_{0} ; \gamma$ denotes the nonlinear coefficient of the waveguide, which is defined as $\gamma=$ $n_{2} k_{0} / a_{\mathrm{eff}}+i \beta_{\mathrm{TPA}} / a_{\mathrm{eff}} ; n_{2}$ is the Kerr coefficient; the parameter $k_{0}$ is the wave vector; $a_{\text {eff }}$ is the effective area of the silicon waveguide; and $\beta_{\mathrm{TPA}}$ is the two-photon absorption (TPA) parameter. The influence of the FCA is from the nonlinear loss $\alpha_{f}=\sigma N_{c}(z, t)$, where $N_{c}(z, t)$ is the density of free carriers. The density of free-carrier $N_{c}(z, t)$ can be obtained by solving the following equation [7]:

$$
\frac{\partial N_{c}(z, t)}{\partial t}=\frac{\beta_{\mathrm{TPA}}}{2 h v_{0}} \frac{|A(z, t)|^{4}}{a_{\mathrm{eff}}^{2}}-\frac{N_{c}(z, t)}{\tau},
$$

where $\tau$ is the effective carrier life time, which is estimated to be about $3 \mathrm{~ns}$ [12], $h$ is Plank constant, and $\nu_{0}$ is the central frequency. All of the parameters above have been considered in our theory model, and the initial injected soliton is assumed to have a hyperbolic secant field profile, which is shown as

$$
A(0, T)=\sqrt{P_{0}} \operatorname{sech}\left(\frac{T}{T_{0}}\right),
$$

where $P_{0}$ is the peak power of the input soliton. The order of input soliton $N$ satisfies the condition of

$$
N^{2}=\frac{\operatorname{Re}(\gamma) P_{0} T_{0}^{2}}{\beta_{2}}
$$

The right-hand side of (1) represents the nonlinear response of the silicon waveguide with the response function $R(t)$, which can be written as

$$
R(t)=\left(1-f_{R}\right) \delta(t)+f_{R} \cdot h_{R}(t)
$$

where the value of $f_{R}=0.043$, which is smaller than that of silica fibers. The function of $h_{R}$ can be written as [15]

$$
h_{R}=\frac{\tau_{1}^{2}+\tau_{2}^{2}}{\tau_{1}^{2} \tau_{2}^{2}} \exp \left(-\frac{t}{\tau_{2}}\right) \cdot \sin \left(\frac{t}{\tau_{1}}\right),
$$

where $\tau_{1}=10 \mathrm{fs}$ and $\tau_{2}=3.03 \mathrm{ps}$.
In order to solve (1) numerically, the equation can be divided to two parts: the linear term and nonlinear term. The linear term includes the effects of linear loss, nonlinear loss, and high order dispersions; here we consider the dispersion coefficient to the sixth order. We can use the split-step Fourier method [11, 16-18] to solve the linear term of (1), while the nonlinear term can be described as follows:

$$
\frac{\partial A}{\partial z}=i \gamma\left(1+\frac{i}{\omega_{0}} \frac{\partial}{\partial t}\right)\left(A R *|A|^{2}\right),
$$

where

$$
R *|A|^{2}=\int_{-\infty}^{t} R\left(t^{\prime}\right)\left|A\left(z, t-t^{\prime}\right)\right|^{2} d t
$$

The time derivative term in (7) can be treated as a perturbation and introduce a new function $B(z, t)$ as

$$
B(z, t)=A(z, t) \cdot \exp \left\lfloor i \gamma R *\left|A_{0}\right|^{2} \cdot\left(z-z_{0}\right)\right\rfloor,
$$

where $z_{0}$ represents the initial position of the propagating femtosecond soliton, considering $z_{0}=0$ in this simulation. From (7) and (9), it can be found that the new function $B(z, t)$ satisfies the following equation:

$$
\frac{\partial B}{\partial z}=i \gamma B \cdot R *\left(\left|B_{0}\right|^{2}+|B|^{2}\right)-\frac{\gamma}{\omega_{0}} \cdot \frac{\partial}{\partial t}\left(B \cdot R *|B|^{2}\right) .
$$

It is possible to obtain $B(z, t)$ through solving (9) using the fourth-order Runge-Kutta integration method [19-21]. The details of calculations using the fourth-order Runge-Kutta method satisfy the following relationship:

$$
B_{k+1}=B_{k}+\frac{h_{0}\left(f_{1}+2 f_{2}+2 f_{3}+f_{4}\right)}{6},
$$

where $h_{0}$ is the step length of the calculation process. $f_{1}, f_{2}$, $f_{3}$, and $f_{4}$ can be written as follows:

$$
\begin{gathered}
f_{1}=f\left(z_{k}, B_{k}\right), \\
f_{2}=f\left(z_{k}+\frac{h_{0}}{2}, B_{k}+\frac{h_{0}}{2} f_{1}\right), \\
f_{3}=f\left(z_{k}+\frac{h_{0}}{2}, B_{k}+\frac{h_{0}}{2} f_{2}\right), \\
f_{4}=f\left(z_{k}+h_{0}, B_{k}+h_{0} f_{3}\right),
\end{gathered}
$$

where $z_{k}$ represents the position of the propagation distance.

Then we can obtain $A(z, t)$ through the relationship between $B(z, t)$ and $A(z, t)$ in (8). Consequently, using the split-step Fourier method (SSFM) and the fourth-order Runge-Kutta integration method, (1) can be solved accurately.

\section{Results and Discussion}

In this simulation, the silicon waveguide used is the straight waveguide. The transverse section of the silicon waveguide is 


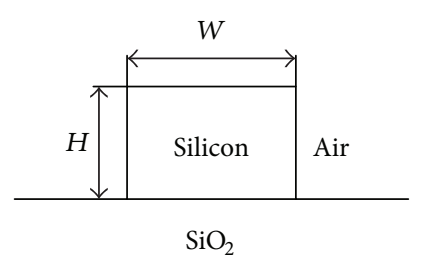

FIGURE 1: Geometry properties of the silicon waveguide. The lower cladding material is $\mathrm{SiO}_{2}$ and the upper cladding material is air. $W=$ $0.7 \mu \mathrm{m}$ denotes the width of the waveguide, and $H=0.5 \mu \mathrm{m}$ is the height of the waveguide.

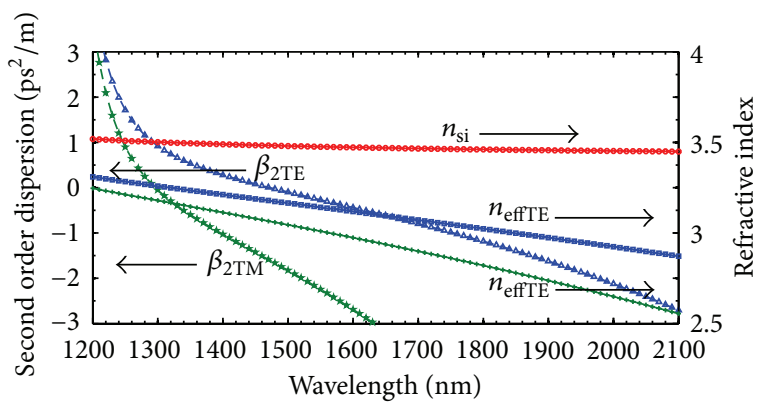

FIgURE 2: Wavelength dependence of $n_{\mathrm{si}}, n_{\mathrm{effTE}}, n_{\mathrm{effTM}}, \beta_{2 \mathrm{TE}}$, and $\beta_{2 \mathrm{TM}}$ for the fundamental TE mode and fundamental TM mode using the waveguide shown in Figure 1 with $W=0.7 \mu \mathrm{m}$ and $H=$ $0.5 \mu \mathrm{m}$.

shown in Figure 1. The dispersion properties of the waveguide play an important role in the process of supercontinuum generation, which can be tailored by choosing the suitable parameters of the waveguide. The zero-dispersion wavelength (ZDWL) must be designed to be around the central wavelength of the pump (1550 nm). The ZDWL of such waveguide can be tailored to fall in this regime with a suitable design. The ZDWL of the transverse electric (TE) mode and transverse magnetic (TM) mode can be tailored to below the pump wavelength for straight waveguide as shown in Figure 1 when both the width and height of the waveguide are close to $0.8 \mu \mathrm{m}$. The width and height of waveguide are assumed as $W=0.7 \mu \mathrm{m}$ and $H=0.5 \mu \mathrm{m}$, respectively.

The wavelength dependence of effective mode index of the TE mode $\left(n_{\text {effTE }}\right)$ and TM mode $\left(n_{\text {effTM }}\right)$ is calculated using the full-vector finite difference mode solver [22] based on the refractive index of silicon waveguide $n_{\mathrm{si}}$. The second dispersion coefficient for TE mode $\left(\beta_{2 \mathrm{TE}}\right)$ and TM mode $\left(\beta_{2 \mathrm{TM}}\right)$ is also calculated as shown in Figure 2. It can be found that $n_{\text {effTE }}$ changes from 3.25 to 2.95 as the wavelength varies from $1200 \mathrm{~nm}$ to $2000 \mathrm{~nm}$ while $n_{\text {effTM }}$ changes from 3.2 to 2.6 in the same wavelength range. The ZDWLs for fundamental TE mode and TM mode of the silicon waveguide are around $1300 \mathrm{~nm}$ and $1500 \mathrm{~nm}$, respectively. In this simulation, the fundamental TE mode can be chosen because the impact of Raman scattering must be considered in the theory model. The higher order dispersion (to the sixth order) parameters of the waveguide for fundamental TE mode at $1550 \mathrm{~nm}$ can also be calculated from the same method, and the dispersion parameters at $1550 \mathrm{~nm}$ are obtained as $\beta_{2}=-0.18 \mathrm{ps}^{2} / \mathrm{m}$, $\beta_{3}=4.0 \times 10^{-3} \mathrm{ps}^{3} / \mathrm{m}, \beta_{4}=-0.75 \times 10^{-5} \mathrm{ps}^{4} / \mathrm{m}, \beta_{5}=$ $0.1 \times 10^{-6} \mathrm{ps}^{5} / \mathrm{m}$, and $\beta_{6}=0.12 \times 10^{-7} \mathrm{ps}^{6} / \mathrm{m}$.

In order to simulate dispersive wave emission and supercontinuum generation in silicon waveguide, we input a femtosecond pulse exciting the fundamental TE mode and propagating in the form of high order soliton. The linear loss coefficient of the waveguide is $\alpha_{l}=0.22 \mathrm{~dB} / \mathrm{cm}$. The TPA coefficient is $\beta_{\mathrm{TPA}}=5 \times 10^{-12} \mathrm{~m} / \mathrm{W}$, the effective area is $a_{\text {eff }}=0.3 \mu \mathrm{m}^{2}$, and the Kerr coefficient is $n_{2}=6 \times 10^{-18} \mathrm{~m}^{2} / \mathrm{W}$ and $\sigma=1.45 \times 10^{-21} \mathrm{~m}^{2}$ for silicon. The input pulse is the femtosecond soliton with the order $N=3$, the pulse peak power is $P_{0}=25 \mathrm{~W}$, and the pulse width is $T_{0}=28.4 \mathrm{fs}$. Correspondingly, the full width of half maximum (FWHM) can be written as

$$
T_{p}=2 \ln (1+\sqrt{2}) T_{0}=1.763 T_{0}=50 \mathrm{fs} .
$$

Compared with the silica waveguide, the Raman scattering plays a minor role in the process of supercontinuum generation in the silicon waveguide. Moreover, the properties of nonlinear loss such as TPA and FCA have impact on the supercontinuum generation in the silicon waveguide, which have been described in [13].

In our simulations, the third order soliton propagates in a $1 \mathrm{~cm}$ long silicon waveguide with the peak power of $25 \mathrm{~W}$. Figure 3 shows the temporal and spectral profiles at the waveguide output. The features seen can be described in terms of soliton fission and dispersive wave emission in time domain while in terms of self-phase modulation and Cherenkov radiation in spectral domain, respectively. It is observed from Figure 3(a) that the single third order soliton evolves into three individual fundamental solitons. Moreover, the dispersive wave emission produces a long tail in time domain as shown in Figure 3(a). Correspondingly, there are some spectral peak structures in spectral domain as shown in Figure 3(b). The spectral peak located at the wavelength $1.3 \mu \mathrm{m}$ is due to the Cherenkov radiation under the condition of phase matching while other spectral peak features are the result of the soliton fission.

In order to accurately explain the supercontinuum generation in silicon waveguide, the temporal and spectral evolution along the waveguide is given in Figure 4 with the same parameters as shown in Figure 3. We first note that the initial stage of propagation is dominated by approximately symmetrical spectral broadening, most of which occurs in the first $1.5 \mathrm{~mm}$. In this process, the effect of self-phase modulation plays a dominated role. Meanwhile, strong temporal compression also occurs over this range. However, with the propagation distance increasing, the spectral broadening becomes greatly asymmetric with the development of distinct spectral peaks on both the short- and long-wavelength sides of the input pump, which is clearly shown in Figure 4(b). As shown in Figure 4(a), when the propagation distance is over $2 \mathrm{~mm}$, the soliton fission effect begins to occur. The full width at half maximum (FWHM) of the spectrum at the distance of $2 \mathrm{~mm}$ is over $100 \mathrm{~nm}$. The dispersive wave emission generates at the distance of $5 \mathrm{~mm}$, which is associated with the development of stable distinct spectral 


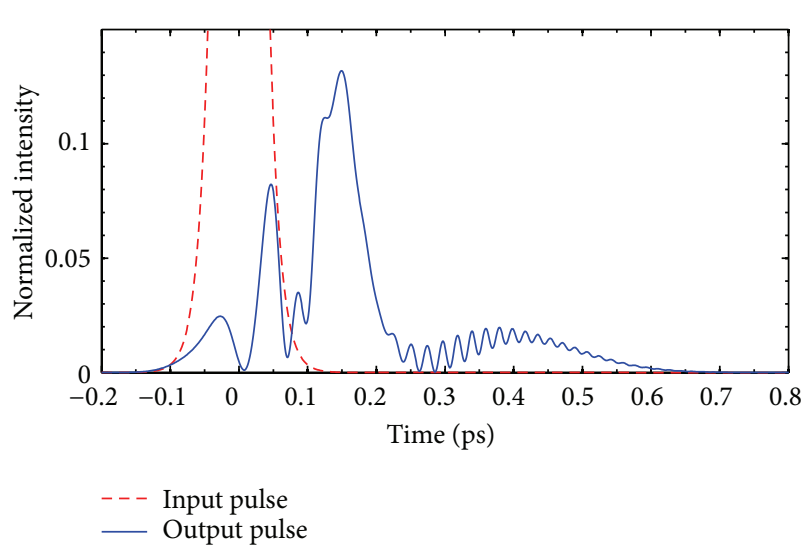

(a)

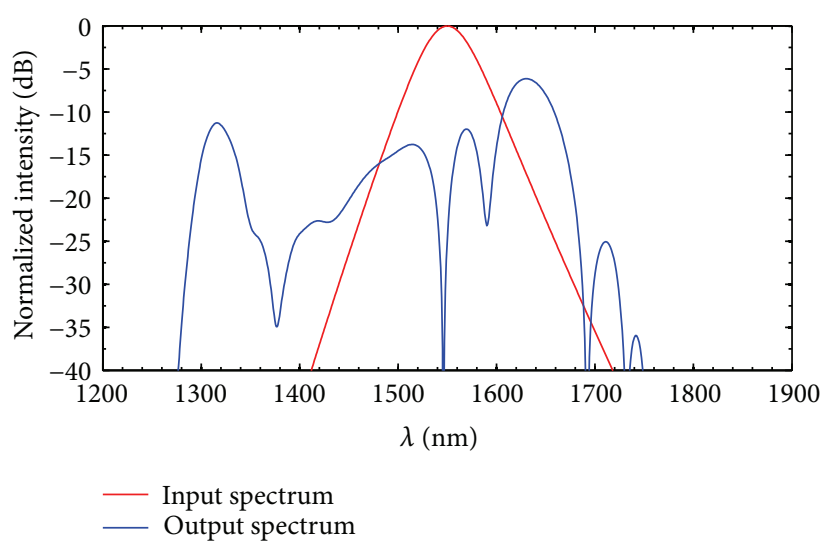

(b)

FiguRE 3: (a) Input and output temporal properties obtained from propagating the $50 \mathrm{fs}$ (FWHM), $25 \mathrm{~W}$ peak power input hyperbolic secant soliton. (b) Input and output spectral properties obtained from propagating the $50 \mathrm{fs}$ (FWHM), $25 \mathrm{~W}$ peak power input hyperbolic secant soliton. The soliton order is $N=3$ and waveguide length is $1 \mathrm{~cm}$.

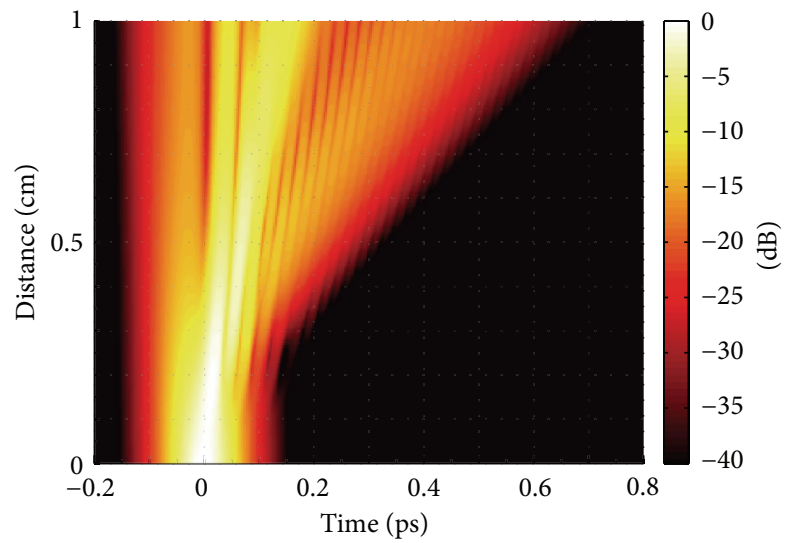

(a)

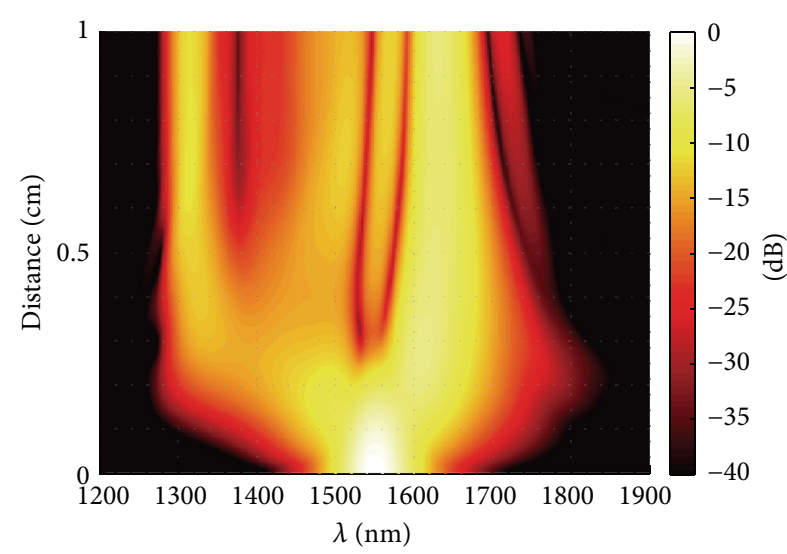

(b)

FIGURE 4: Results from numerical simulations showing (a) temporal and (b) spectral evolution for $1 \mathrm{~cm}$ long silicon waveguide. The input soliton is $50 \mathrm{fs}$ (FWHM), $25 \mathrm{~W}$ peak power hyperbolic secant soliton with order $N=3$.

peak in spectral domain called the Cherenkov radiation. Here we define the full width at half maximum (FWHM) of the pulse and spectrum as the pulse width and spectral width, respectively. The pulse width and spectral width at different distance of the waveguide can be seen clearly in Figure 5.

The Cherenkov radiation is emitted in the form of dispersive wave at a frequency underlying phase matching condition. The frequency detuning of Cherenkov radiation is given by [12]

$$
\Omega_{d} \approx-\frac{3 \beta_{2}}{\beta_{3}}+\frac{\gamma P_{s} \beta_{3}}{3 \beta_{2}^{2}}
$$

where $P_{s}$ is the peak power of the fundamental soliton perturbed by the third order dispersion coefficient. Generally, in the absence of losses, $P_{s}=(5 / 3)^{2} P_{0}$, where $P_{0}$ is the peak power of the input soliton. However, the linear loss and nonlinear loss reduce the value of $P_{s}$ along the waveguide. We assume that $P_{s} \approx P_{0}$ in this simulation. The frequency detuning of Cherenkov radiation is determined by the high order dispersions and input peak power of solitons. The relationship between the frequency of Cherenkov radiation and input peak power is presented in Figure 6; when the input peak power of soliton changes from $10 \mathrm{~W}$ to $70 \mathrm{~W}$, the frequency of dispersive wave emission varies from $170 \mathrm{THz}$ to more than $370 \mathrm{THz}$. It is also known that the frequency of dispersive wave emission can be changed when pumping normal dispersion zone and abnormal dispersion zone, which is described by (17).

The results of temporal and spectral evolution by inputting high order soliton with $N=6$ and the peak power of soliton which is $50 \mathrm{~W}$ are obtained as shown in Figure 7. It is observed that soliton fission occurs in the process of pulse evolution and the broadband dispersive wave is generated as shown in Figure 7(a). In this process, the number of solitons generated by the soliton fission is 6 , which is the same with the order of soliton. Figure 7(b) shows the bandwidth of the supercontinuum is becoming to over $500 \mathrm{~nm}$, which 


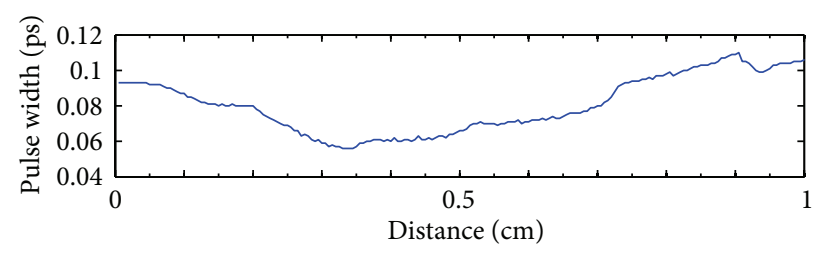

(a)

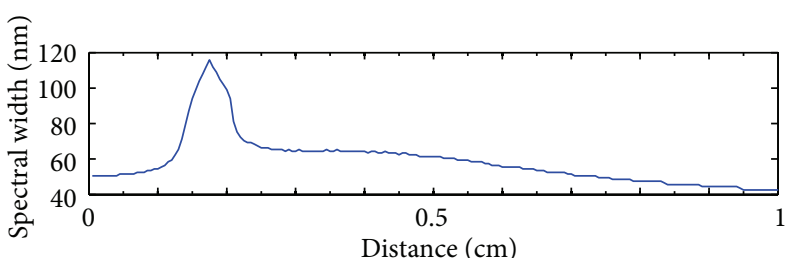

(b)

FIGURE 5: Results from numerical simulations showing (a) variation of pulse width along the silicon waveguide and (b) variation of spectral width along the silicon waveguide.

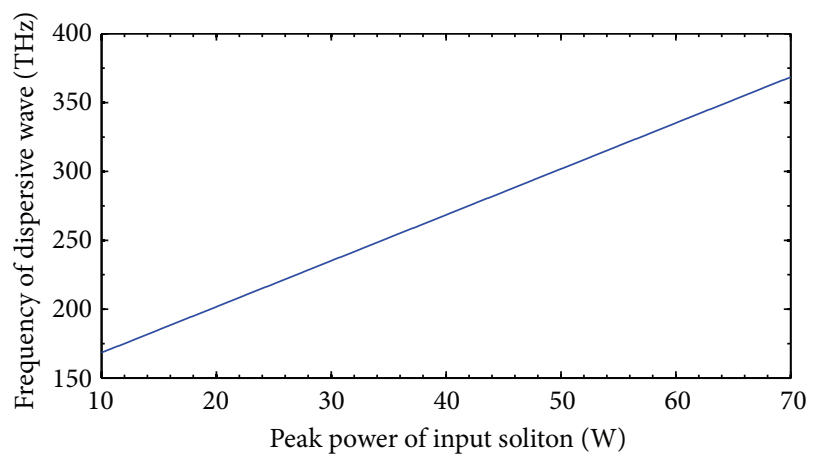

FIGURE 6: Frequency of dispersive wave emission as a function of inputting peak power of the soliton. The relative parameters are the same as used in Figure 4.

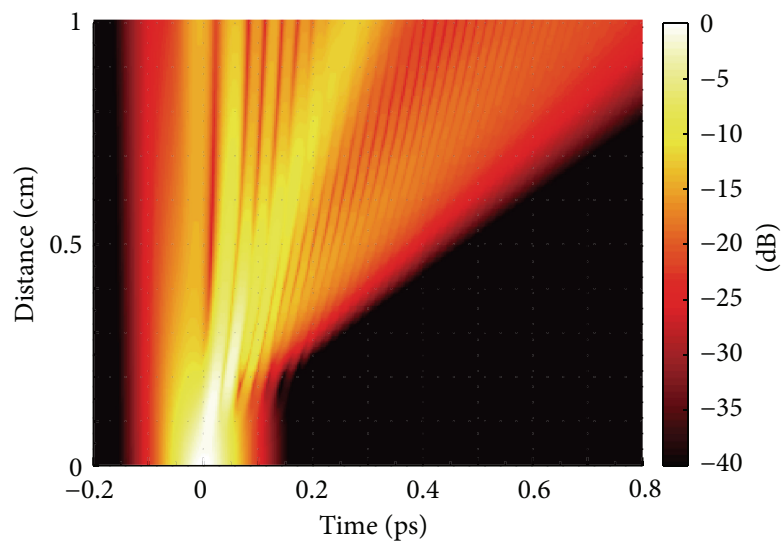

(a)

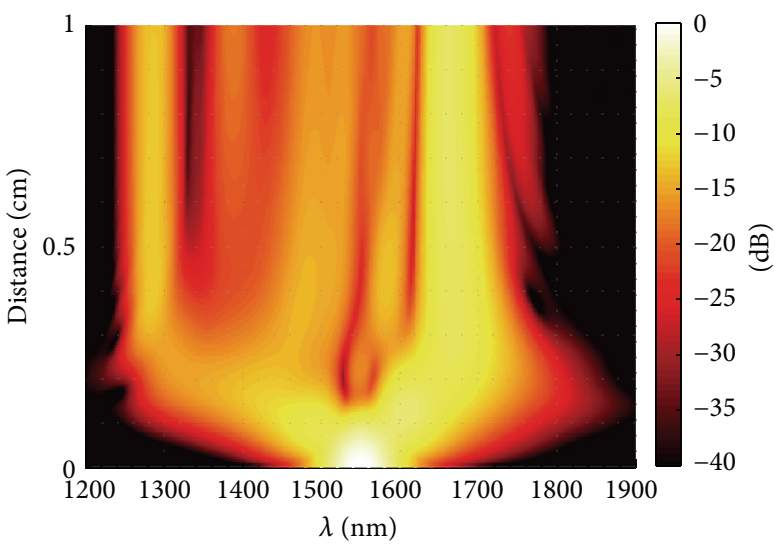

(b)

FIGURE 7: Results from numerical simulations showing (a) temporal and (b) spectral evolution for $1 \mathrm{~cm}$ long silicon waveguide. The input soliton is $50 \mathrm{fs}$ (FWHM), $50 \mathrm{~W}$ peak power hyperbolic secant soliton with order $N=6$.

is broader than that generated by the third order soliton. It can be also found that the wavelength of Cherenkov radiation is around $1250 \mathrm{~nm}$. This value agrees with the predictions of (12). Compared with the supercontinuum generation in silica fibers, the effect of stimulated Raman scattering in silicon waveguide plays a minor role. The effect of TPA and FCA must be considered in the process of supercontinuum generation in silicon waveguide. Figure 8(a) shows the temporal evolution along the waveguide without considering the effect of TPA; the generated dispersive wave emission is more continuous and the efficiency is higher than that generated considering TPA. The contrast between the input and output pulse without the effect of TPA is shown in Figure 8(b), in which the intensity of soliton fission is reduced and the intensity of fundamental soliton is nearly $80 \%$ of input intensity. The effect of TPA is not detrimental to the supercontinuum generation process, even though it reduces the supercontinuum bandwidth to some extent. The numerical results of the dispersive wave emission without considering the effect of stimulated Raman scattering (SRS) were also presented. Figure 9(a) shows the temporal evolution along the waveguide without considering the effect of SRS. The contrast between the input and output pulse without the effect of SRS is shown in Figure 9(b). The soliton 


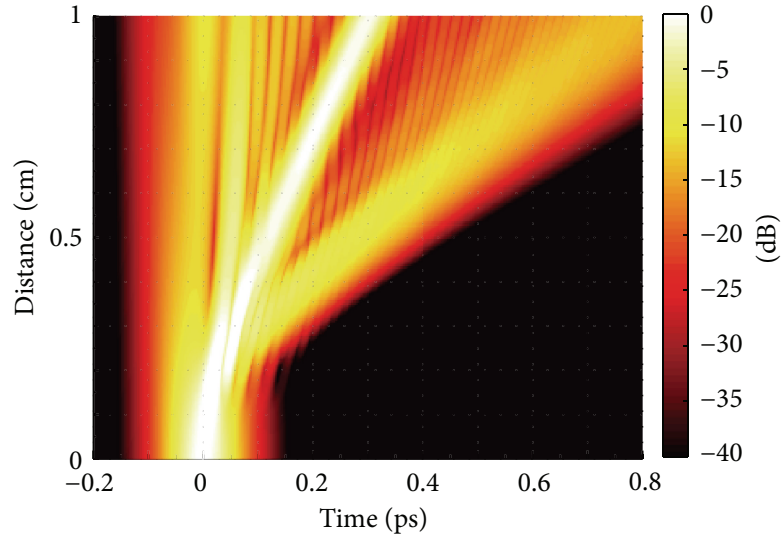

(a)

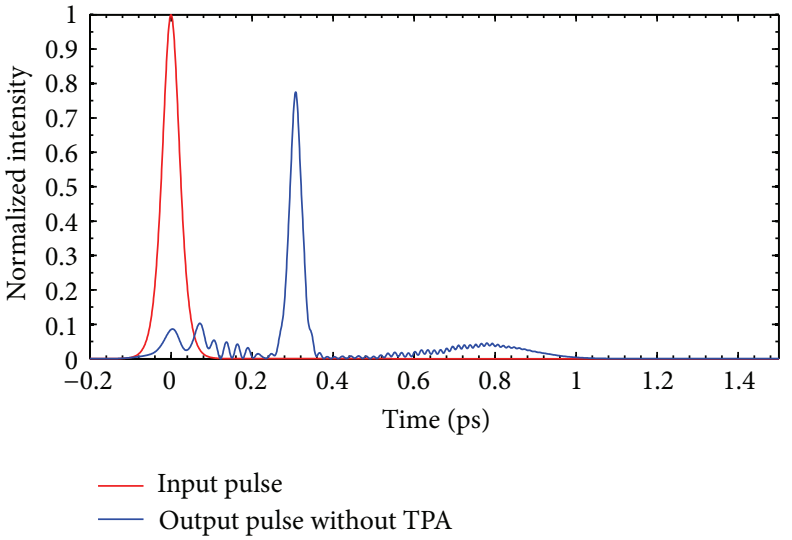

(b)

FIGURE 8: (a) Temporal evolution for the $1 \mathrm{~cm}$ long silicon waveguide without considering the effect of TPA. (b) Contrast of the input pulse and output pulse without considering the effect of TPA. The input soliton is $50 \mathrm{fs}$ (FWHM), $25 \mathrm{~W}$ peak power hyperbolic secant soliton with order $N=3$.

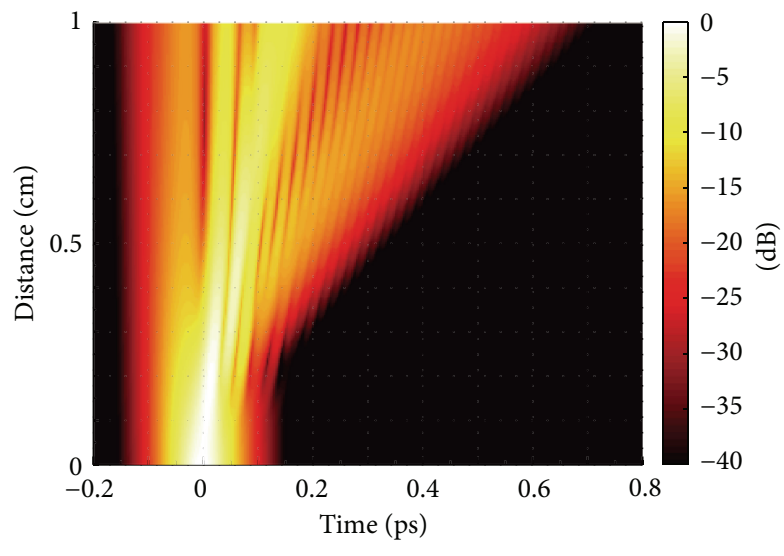

(a)

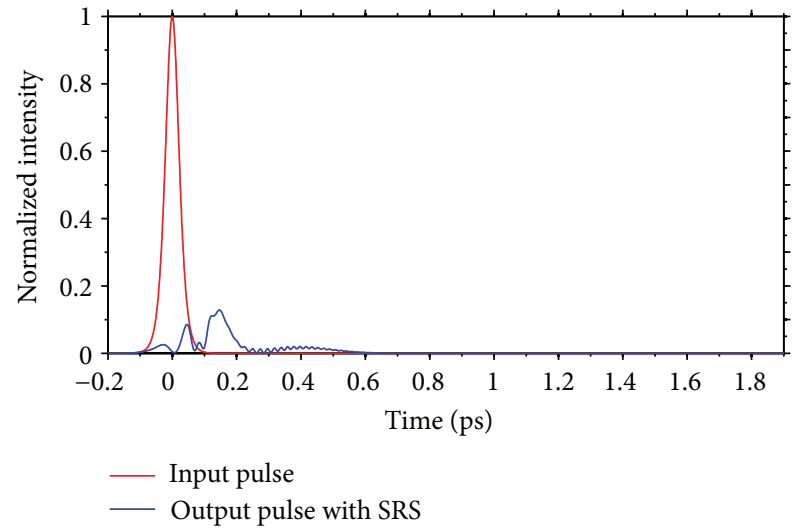

(b)

FIgURE 9: (a) Temporal evolution for the $1 \mathrm{~cm}$ long silicon waveguide without considering the effect of stimulated Raman scattering. (b) Contrast of the input pulse and output pulse without considering the effect of stimulated Raman scattering. The input soliton is $50 \mathrm{fs}$ (FWHM), $25 \mathrm{~W}$ peak power hyperbolic secant soliton with order $N=3$.

fission effect is weak without the role of SRS, which gives rise to the reduction of the number of the peak in time domain.

\section{Conclusions}

Based on solving the generalized nonlinear Schrödinger equation in the silicon waveguide through the SSFM and fourth-order Runge-Kutta integration method, the dispersive wave emission and supercontinuum generation are investigated in silicon waveguide. The numerical results show that the efficient dispersive wave emission can be generated by propagating femtosecond solitons in silicon waveguide, which plays an important role in the process of the supercontinuum generation with the form of Cherenkov radiation, and it is also shown that the high order low-energy solitons and short waveguides are efficient for the dispersive wave emission, and the dominated impacts on the generation of the dispersive wave emission are the high order dispersions and the peak power of input soliton.

\section{Conflict of Interests}

The author declares that there is no conflict of interests regarding the publication of this paper.

\section{Acknowledgment}

This work was supported by the National Natural Science Foundation of China under Grant no. 61275134. 


\section{References}

[1] J. M. Dudley and J. R. Taylor, "Ten years of nonlinear optics in photonic crystal fibre," Nature Photonics, vol. 3, no. 2, pp. 85-90, 2009.

[2] P. L. Baldeck and R. R. Alfano, "Intensity effects on the stimulated four photon spectra generated by picosecond pulses in optical fibers," Journal of Lightwave Technology, vol. 5, no. 12, pp. 1712-1715, 1987.

[3] J. Herrmann, U. Griebner, N. Zhavoronkov et al., "Experimental evidence for supercontinuum generation by fission of higherorder solitons in photonic fibers," Physical Review Letters, vol. 88, no. 17, Article ID 173901, 2002.

[4] A. L. Gaeta, "Nonlinear propagation and continuum generation in microstructured optical fibers," Optics Letters, vol. 27, no. 11, pp. 924-926, 2002.

[5] A. Demircan and U. Bandelow, "Analysis of the interplay between soliton fission and modulation instability in supercontinuum generation," Applied Physics B: Lasers and Optics, vol. 86, no. 1, pp. 31-39, 2007.

[6] M. A. Foster, J. M. Dudley, B. Kibler et al., "Nonlinear pulse propagation and supercontinuum generation in photonic nanowires: experiment and simulation," Applied Physics B: Lasers and Optics, vol. 81, no. 2-3, pp. 363-367, 2005.

[7] Q. Lin, O. J. Painter, and G. P. Agrawal, "Nonlinear optical phenomena in silicon waveguides: modeling and applications," Optics Express, vol. 15, no. 25, pp. 16604-16644, 2007.

[8] M. Zhu, H. Liu, X. Li et al., "Ultrabroadband flat dispersion tailoring of dual-slot silicon waveguides," Optics Express, vol. 20, pp. 15899-15907, 2012.

[9] L. Yin and G. P. Agrawal, "Impact of two-photon absorption on self-phase modulation in silicon waveguides," Optics Letters, vol. 32, no. 14, pp. 2031-2033, 2007.

[10] Z. Wang, H. Liu, N. Huang, Q. Sun, J. Wen, and X. Li, "Influence of three-photon absorption on Mid-infrared crossphase modulation in silicon-on-sapphire waveguides," Optics Express, vol. 21, pp. 1840-1848, 2013.

[11] X. Li, Z. Wang, and H. Liu, "Optimizing initial chirp for efficient femtosecond wavelength conversion in silicon waveguide by split-step Fourier method," Applied Mathematics and Computation, vol. 218, pp. 11970-11975, 2012.

[12] L. Yin, Q. Lin, and G. P. Agrawal, "Soliton fission and supercontinuum generation in silicon waveguides," Optics Letters, vol. 32, no. 4, pp. 391-393, 2007.

[13] I.-W. Hsieh, X. Chen, X. Liu et al., "Supercontinuum generation in silicon photonic wires," Optics Express, vol. 15, no. 23, pp. 15242-15249, 2007.

[14] J. Wen, H. Liu, N. Huang, Q. Sun, and W. Zhao, "Influence of the initial chirp on the supercontinuum generation in siliconon-insulator waveguide," Applied Physics B: Lasers and Optics, vol. 104, no. 4, pp. 867-871, 2011.

[15] K. J. Blow and D. Wood, "Theoretical description of transient stimulated Raman scattering in optical fibers," IEEE Journal of Quantum Electronics, vol. 25, no. 12, pp. 2665-2673, 1989.

[16] H. Wang, "Numerical studies on the split-step finite difference method for nonlinear Schrödinger equations," Applied Mathematics and Computation, vol. 170, no. 1, pp. 17-35, 2005.

[17] X. Xiangming and T. R. Taha, "Parallel split-step fourier methods for nonlinear Schrodinger-type equations," Journal of Mathematical Modelling and Algorithms, vol. 2, pp. 185-201, 2003.
[18] G. M. Muslu and H. A. Erbay, "Higher-order split-step Fourier schemes for the generalized nonlinear Schrödinger equation," Mathematics and Computers in Simulation, vol. 67, no. 6, pp. 581-595, 2005.

[19] S. Zhang, Z. Deng, and W. Li, "A precise Runge-Kutta integration and its application for solving nonlinear dynamical systems," Applied Mathematics and Computation, vol. 184, no. 2, pp. 496-502, 2007.

[20] M. Z. Liu, S. F. Ma, and Z. W. Yang, "Stability analysis of RungeKutta methods for unbounded retarded differential equations with piecewise continuous arguments," Applied Mathematics and Computation, vol. 191, no. 1, pp. 57-66, 2007.

[21] B. S. Attili, K. Furati, and M. I. Syam, "An efficient implicit Runge-Kutta method for second order systems," Applied Mathematics and Computation, vol. 178, no. 2, pp. 229-238, 2006.

[22] T. E. Murphy, software, http://www.photonics.umd.edu/. 


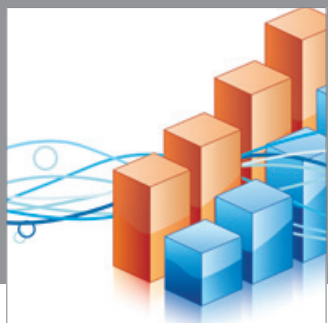

Advances in

Operations Research

mansans

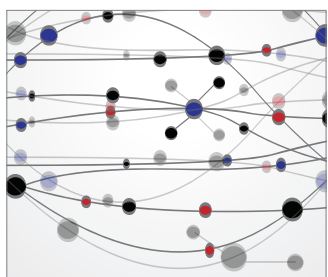

The Scientific World Journal
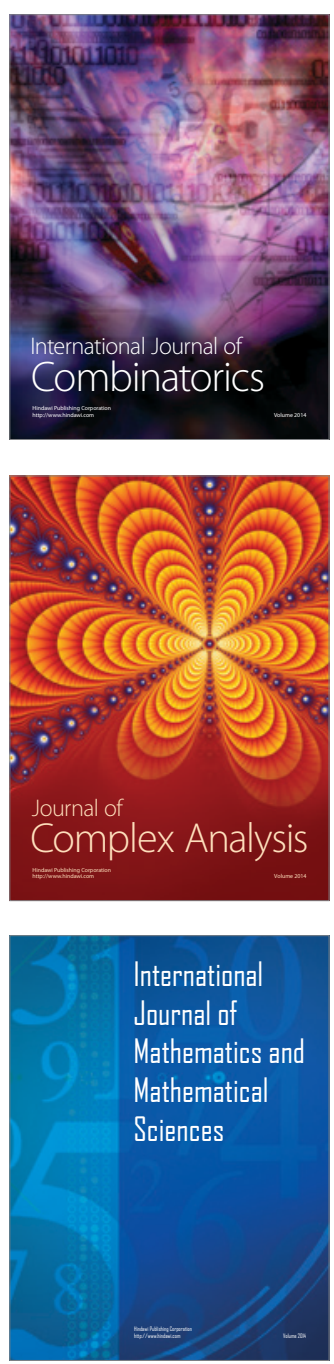
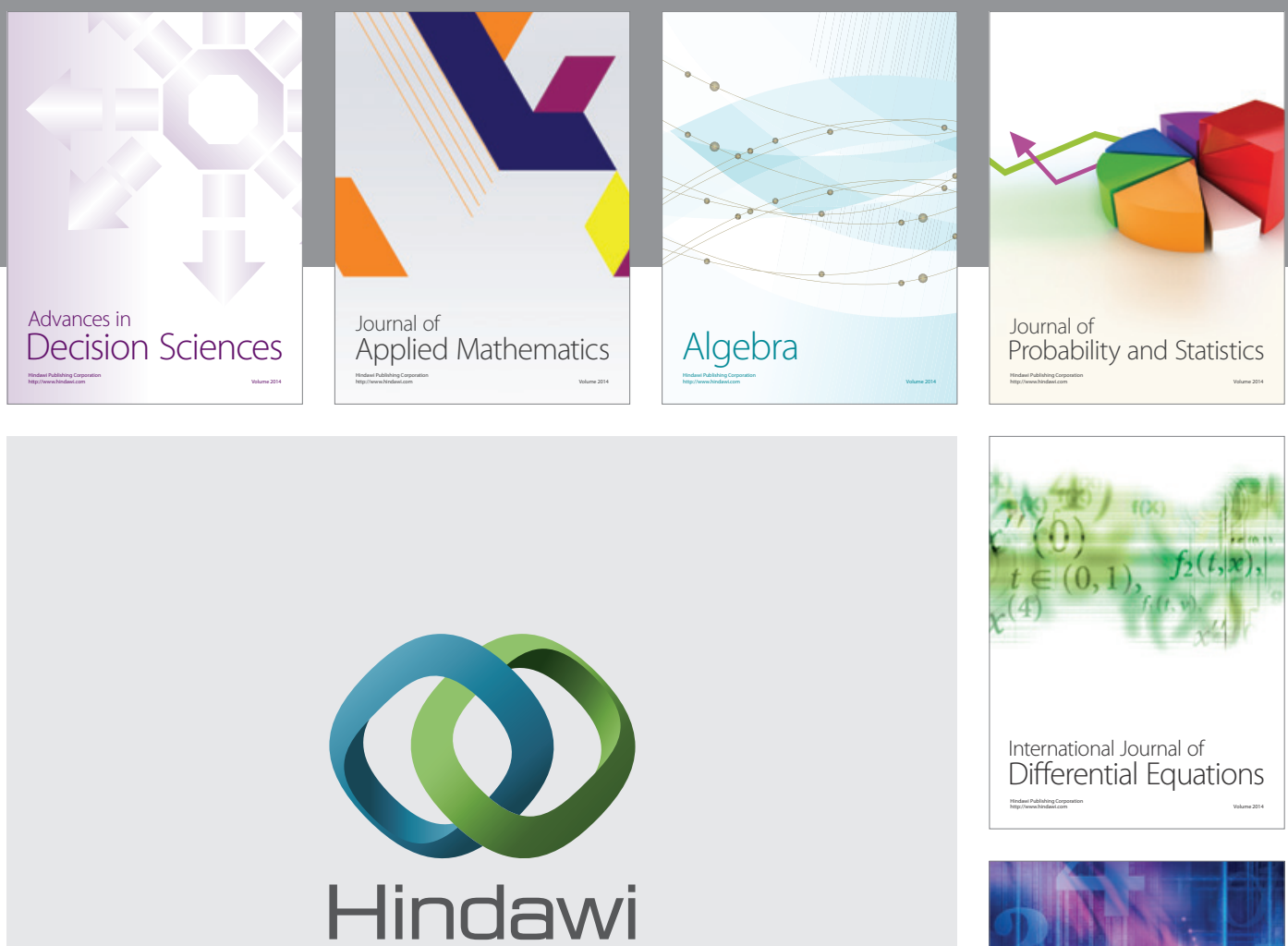

Submit your manuscripts at http://www.hindawi.com
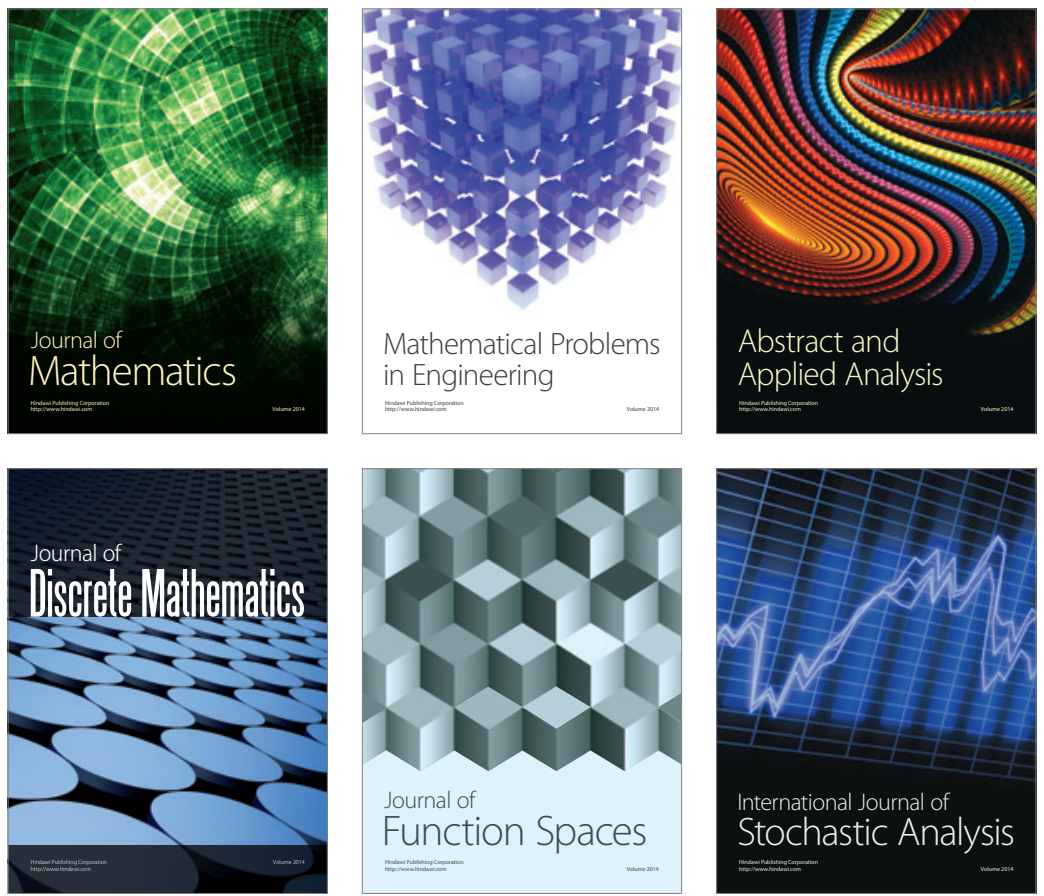

Journal of

Function Spaces

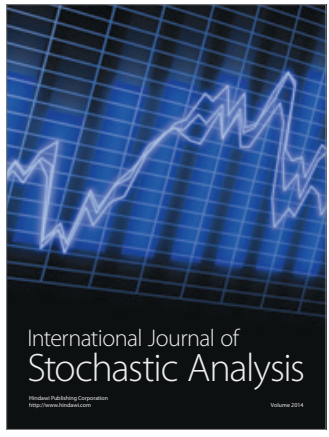

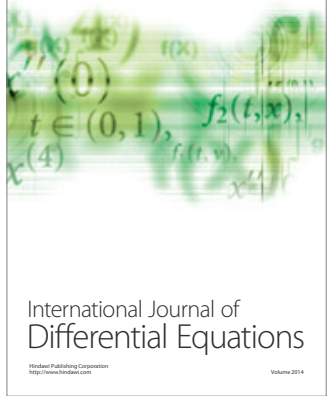
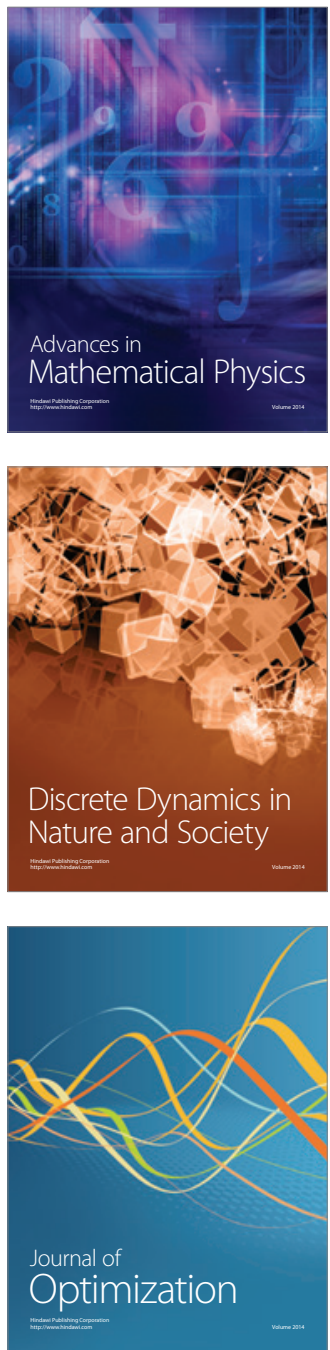